\title{
Growth modification of seeded calcite using carboxylic acids: Atomistic simulations
}

\author{
Ulrich Aschauer $^{\mathrm{a}, *, 1}$, Dino Spagnoli ${ }^{\mathrm{b}, 2}$, Paul Bowen $^{\mathrm{a}}$, Stephen C. Parker ${ }^{\mathrm{b}}$ \\ ${ }^{a}$ Laboratoire de technologie des poudres (LTP), IMX/STI/EPFL, Station 12, 1015 Lausanne, Switzerland \\ ${ }^{\mathrm{b}}$ Department of Chemistry, University of Bath, Claverton Down, Bath BA2 7AY, UK
}

\section{A R T I C L E I N F O}

\section{Article history:}

Received 3 February 2010

Accepted 24 February 2010

Available online 1 March 2010

\section{Keywords:}

Calcium carbonate

Calcite

Polycarboxylic acids

Atomistic simulation

Adsorption

\begin{abstract}
A B S T R A C T
Molecular dynamics simulations were used to investigate possible explanations for experimentally observed differences in the growth modification of calcite particles by two organic additives, polyacrylic acid (PAA) and polyaspartic acid (p-ASP). The more rigid backbone of p-ASP was found to inhibit the formation of stable complexes with counter-ions in solution, resulting in a higher availability of p-ASP compared to PAA for surface adsorption. Furthermore the presence of nitrogen on the p-ASP backbone yields favorable electrostatic interactions with the surface, resulting in negative adsorption energies, in an upright (brush conformation). This leads to a more rapid binding and longer residence times at calcite surfaces compared to PAA, which adsorbed in a flat (pancake) configuration with positive adsorption energies. The PAA adsorption occurring despite this positive energy difference can be attributed to the disruption of the ordered water layer seen in the simulations and hence a significant entropic contribution to the adsorption free energy. These findings help explain the stronger inhibiting effect on calcite growth observed by p-ASP compared to PAA and can be used as guidelines in the design of additives leading to even more marked growth modifying effects.
\end{abstract}

(C) 2010 Elsevier Inc. All rights reserved.

\section{Introduction}

Calcite is a calcium carbonate $\left(\mathrm{CaCO}_{3}\right)$ polymorph important in many fields of science and technology ranging from being a component in animal skeletons [1-3], applications as fillers in polymers [4] or pigments in paints, and use as a catalysts [5] or substrates in chemical engineering. For many technological applications a precise control over the particle size, morphology and specific surface area would be highly desirable. One way to gain this level of control is the use of growth modifying additives during the precipitation of calcite from the aqueous phase. It has been shown in the past that the adsorption of many types of polymers such as hydrophilic block copolymers [6,7], polycarboxylic acids [8-11], phosphonates [12,13] and polyamino acids [14-17] during precipitation or dissolution can modify calcite particle size and morphology. It was also shown that polycarboxylic acids can promote a very particular growth mechanisms [18], during which

\footnotetext{
* Corresponding author. Address. Princeton University, Chemistry Department, Frick Laboratory, Room 213, Princeton, NJ 08544, USA. Fax: +1 6092586746.

E-mail addresses: uli.aschauer@epfl.ch, aschauer@princeton.edu (U. Aschauer), dino@ivec.org (D. Spagnoli), paul.bowen@epfl.ch (P. Bowen), s.c.parker@bath.ac.uk (S.C. Parker).

1 Present address: Department of Chemistry, Princeton University, Princeton, NJ 08544, USA.

2 Present address: Nanochemistry Research Institute, Department of Chemistry, Curtin University of Technology, Perth, WA 6845, Australia.
}

agglomeration of small primary particles results in a very high overall specific surface area. High specific surface area materials are of great interest in catalysis and for pharmaceutical applications such as drug-delivery.

In a separate publication our group reported experimental results of the effects of the polycarboxylate, polyacrylic acid (PAA), as well as the polypeptide, polyaspartic acid (p-ASP), on the precipitation of calcite [19]. It was found that the specific surface area and thus the degree of nanostructuration are more pronounced in the presence of p-ASP than PAA. This suggests that p-ASP must have a stronger interaction with calcite surfaces than PAA, which cannot be readily explained from experiment alone. Both polymers carry the same functional carboxylic acid group, PAA having at experimental conditions ( $\mathrm{pH} 8-9$ ) only a slightly lower fraction of deprotonated carboxylic acid groups (0.815) [20] than the fully deprotonated p-ASP. The difference in interaction is thus very difficult to characterize experimentally and computational methods can be of great help to gain insights and better understand experimental observations.

Molecular modeling studies on calcite have been carried out for nearly two decades. The equilibrium morphology and its variation due to ionic species has been calculated in vacuum [21] showing the morphological dominance of the $(10 \overline{1} 4)$ face in the absence of extrinsic ions. Growth inhibition by diphosphonates has been studied in vacuum [22] and adsorption was found to be stronger at steps than on the flat surface, giving a possible explanation for the growth 
modifying mechanism. Monophosphonate additives were shown to strongly bind to steps in vacuum with the predominant driving force being electrostatic [23], Changes in the step-edge free energy due to selective adsorption of amino acids [24] were shown to govern crystal growth in addition to stereochemical effects. Adsorption of several organic additives (hydroxyl, carbonyl and amine functional groups) at hydrated calcite steps as well as the flat (1014) surface was investigated [25] indicating a strong binding of the hydroxyl and carbonyl groups to step edges, whereas the amine groups bound only weakly. The authors concluded that based on their results carboxylic acids should have good growth inhibiting capacities. Interaction of stearic acid with calcite surfaces has been simulated [26] and it was predicted that the degree of ionization of the polymer is very important as different surfaces are favorably stabilized at different degrees of ionization, resulting in different morphologies. Polyaspartate interaction with step edges has been modeled [17] and it was found that at short chain lengths the polymer preferentially binds to acute steps whereas longer molecules prefer obtuse steps. The reason for this behavior was attributed to be the balance between the energy required to dehydrate the step and the adsorption energy.

Based on the existing literature it is impossible to predict the origin of the difference between PAA and p-ASP as most often only one class of additive was studied therefore polycarboxylates and polyamino acids never being directly compared. Also many studies have been made in vacuum and not in water which also needs to be addressed. This study uses molecular dynamics simulations to study PAA and p-ASP adsorption on calcite surfaces from an aqueous environment, focusing on the more abundant $\beta$-form of p-ASP [27]. Results obtained from this study will help in understanding experiments on the atomistic level and aid in the ultimate goal, which is to produce tailor made calcite particles of known particle size and morphology for high specific surface area applications.

\section{Computational method}

All simulations have been treated using the Born model for solids [28], which describes interatomic interactions as a combination of long-range electrostatic and short-range interactions. The shortrange interactions include electron-cloud overlap and van der Waals attractive terms, and for the carbonate ion also many-body terms in order to correctly reproduce the bond geometry. The quality of our simulations to provide a realistic representation of the system in question relies solely on the interatomic parameters used to describe the interactions between the different ionic species. Polarizability of the carbonate and water oxygen atoms is taken into account by a core-shell model as devised by Dick and Overhauser [29]. In this model the oxygen is split into two particles attached to each other by a harmonic spring potential and carrying together the overall charge of the ion. Pavese et al. first derived the calcite potential parameters used in this study [30-31] which have since been successfully applied to the accurate representations of calcite surfaces [32-37]. These potentials were used to construct flat and stepped surfaces starting from the crystallographic calcite unit-cell using the computer code METADISE [38].

The water potential is that of de Leeuw and Parker [39] with the added hydrogen bonding modification of Kerisit and Parker [40]. Calcite-water and sodium ion-water interactions are described using the potentials of Kerisit and Parker [40] and Spagnoli et al. [41]. The intra-additives and additive/water term were taken from the DREIDING [42] organic force-field together with the potentials derived by Duffy and Harding [26] for stearic acid, which has the same functional groups, in order to describe the interaction between the mineral and the polymer. The potential for sodium-carboxylate interaction uses a scaled [43] version of the calcium- carboxylate potential. Charge distributions within the completely deprotonated 10 monomer additives PAA and $\beta$-p-ASP was calculated using the GAUSSIAN [44] code, using Mulliken [45] charges. In order to reduce the computational effort, the masses and charges of the hydrogen atoms were summed to their bonded heavier atoms. A file containing all interatomic interactions used in this work is available as supporting information. It should be noted that recently a more detailed approach to assembling a suitable force-field has been published [46], the foundations are however the same and results are expected not to vary much for calculations using this new potential.

All molecular dynamics simulations were performed using the DL_POLY 2 [47] code applying the Verlet Leap Frog algorithm with a timestep of $0.2 \mathrm{fs}$ for shell masses of $0.2 \mathrm{amu}$. For equilibration the NPT (variable volume) ensemble was used whereas production was carried out within the NVT (constant volume) ensemble by applying Nose-Hoover thermostats and Hoover barostats with relaxation times of $0.5 \mathrm{ps}$ as appropriate. The interatomic interactions were calculated up to a cutoff of $8 \AA$ which was also used as the real-space cutoff in the smooth particle mesh Ewald (SPME) algorithm used for the calculation of the long-range electrostatic interactions.

\section{Results and discussion}

In the first simulation we attempted to study adsorption of the additives on the morphology dominating flat (1014) surface of calcite (surface area $24 \times 24 \AA$ ). The deprotonated additives (10 negatively charged carboxylate groups) with the corresponding number of charge balancing counter-ions $\left(\mathrm{Na}^{+}\right.$or $\left.\mathrm{Ca}^{2+}\right)$ was placed in a $40 \AA$ thick water layer at about $15 \AA$ above a calcite slab of thickness $15 \AA$. During the equilibration, which was performed in the NPT ensemble for $0.5 \mathrm{~ns}$ the water molecules were allowed to explore all of the configurational space whereas the additive, counter-ions and calcite surface were fixed in position so as to retain this setup for the start of the production run. The production run was carried out for $1 \mathrm{~ns}$ in the NVT ensemble with all atoms mobile. Adsorption of p-ASP and PAA did not occur. In both cases the additive remained in a layer about $4 \AA$ from the calcite surface during the whole simulation time. This may be related to two phenomena, the first of which is complex formation of the additive with the counter-ions in solution. Polycarboxylic acids are known to strongly complex $\mathrm{Ca}^{2+}$ and to lesser extent $\mathrm{Na}^{+}$ions [48-49], which could result in a reduction of the capacity of the additive to bind to the surface. The second reason may be the highly layered structure of water found above a calcite surface with regions of alternating low and high water density $[40,50]$. As the additive backbones are hydrophobic, the molecules will have a tendency to stay in low density water regions, which are known from both experiment [50] and simulations [40] to be located roughly at $2.8 \AA$ and $3.9 \AA$ from the surface.

In order to test the first hypothesis, simulations of complex formation in solution without presence of calcite were carried out and the normalized area below each curve equals (1) radial distribution functions (RDF) analyzed (Fig. 1). As it can be seen the water structure around the calcium ions (dark lines) is the same for both additives (PAA solid line and p-ASP dashed line). The structure in presence of carboxylic acid groups however is very different, the peak of the distribution for PAA being collocated with the first coordination shell of the calcite-water structure whereas for p-ASP it is collocated with the second peak only. This means that PAA forms strong inner sphere complexes with the counter-ions and p-ASP forms weaker outer sphere complexes only. The lifetime of inner sphere complexes is expected to be longer than that of outer sphere complexes, because of the stronger electrostatic interactions. This 


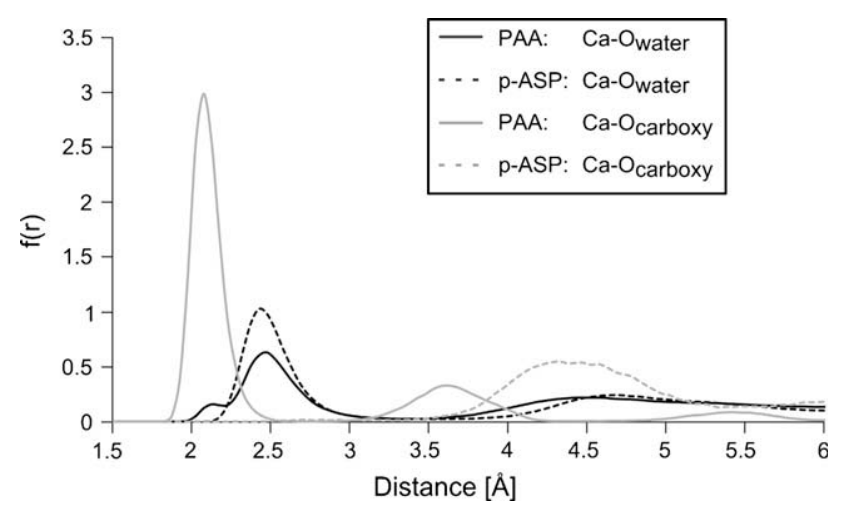

Fig. 1. Normalized radial distribution functions of the $\mathrm{Ca}-\mathrm{O}_{\text {water }}$ (dark lines) and $\mathrm{Ca}-\mathrm{O}_{\text {carboxy }}$ (light lines) distances for the two additives PAA (solid lines) and p-ASP (dashed lines).

was also observed in the simulation trajectories where the p-ASP complex continuously broke and reformed with different carboxylic acid groups, whereas the PAA complex remained in its configuration for the whole simulation time of 1 ns. These very stable coiled configurations were also observed in previous calculations of polyacrylates with calcium counter-ions [51]. The same calculations were run for sodium counter-ions (Fig. 2), where inner sphere complex formation was observed for both additives, however to a much lesser extent for p-ASP. This can be explained by the less restricted complex structure for sodium where only a single carboxy group per ion is required. These results, besides predicting a higher surface adsorption activity for $\mathrm{p}$-ASP, also give a first hint that the backbone, which due to the peptide bond is more rigid for $\mathrm{p}$-ASP, restricts the configuration this molecule can adopt.

The second hypothesis, that a large energy barrier is hindering adsorption, was tested by creating an additional driving force for adsorption by removing $\mathrm{CO}_{3}^{2-}$ groups from the surface. This creates a positively charged defect at the surface, which will interact with the deprotonated carboxy groups. Continuum electrostatic calculations [52] recently showed strong electrostatic interactions of this kind to play an important role for the adsorption of peptides on calcite surfaces. Creating surface vacancies also allows us to remove counter-ions from the simulation thus also eliminating complex formation. Although counter-ions are clearly present in experiment, their residence time with carboxylic acid groups is known to be on the order of 530 ps (water 80 ps) [53]. The kinetics may therefore be slightly affected by the absence of counter-ions but the overall process should be the same. Simulations were run

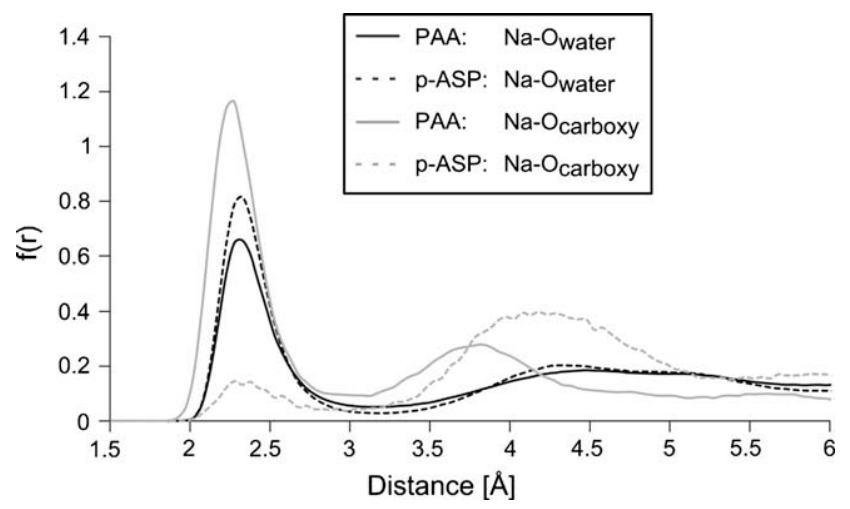

Fig. 2. Normalized radial distribution functions of the $\mathrm{Na}-\mathrm{O}_{\text {water }}$ (dark lines) and $\mathrm{Na}-\mathrm{O}_{\text {carboxy }}$ (light lines) distances for the two additives PAA (solid lines) and p-ASP (dashed lines). with an increasing number of carbonates removed, with adsorption occurring only when three or more carbonate vacancies exist at the surface. This shows that the presence of charged defects at the surface helps to overcome the energy barrier resulting from the structure of coordinated water at the surface (i.e. dehydration energy). In fact when pushed onto the surface, the additives were even found to desorb without presence of charged defects. This implies that without presence of charged defects the hydration of the surface is energetically more favorable than the adsorption of the additive and that the presence of an additional electrostatic component to the adsorption energy is required for adsorption to occur. This aspect is especially important, as it has not been present in many of the previous simulations of polymer adsorption that were often carried out in vacuum. Consequently additives can be assumed to bind rapidly to these defect sites, stoichiometric parts of the surface being covered by the additive at a later stage and at sufficient additive concentration.

Experimental observations of calcite surfaces [12,54] show a high density of steps, where carbonate dissolution at the $\mathrm{pH}$ of interest (around nine) is more favorable [55], which is also predicted by theory [56]. Even stable surfaces such as the (1014) surface have been shown to reconstruct in moist air via pit formation and film growth [57], making it reasonable to consider defective stepped surfaces in our simulations. In order to have an estimate of the number of carbonate groups to be removed, experimental zeta-potential measurements $(-13 \mathrm{mV})$ at low $\mathrm{CO}_{2}$ partial pressure [58] were considered and the surface charge density $\varphi$ calculated via the surface potential $\psi$ as given by [59].

$$
\begin{aligned}
& \psi=\zeta \exp (\kappa d) \\
& \varphi=\sinh \left(\frac{\psi z e}{2 k T}\right) \sqrt{8 k T c_{0}^{*} \varepsilon_{0} \varepsilon}
\end{aligned}
$$

The surface potential is deduced from the zeta potential $\zeta$ by knowing the solvent dependent Debye length [59] $1 / \kappa$ and assuming the zeta potential is measured at a distance $d$ from the surface (here fixed at $0.5 \mathrm{~nm}$ ). The surface charge density depends on the temperature $T$, as well as the charge $z$ and concentration $c_{0}^{*}$ of the ions in solution and the dielectric constant $\varepsilon$ of the solution.

To replicate the experimental zeta potential [58], 4-5 carbonate groups have to be removed per simulation box with a surface measuring about $25 \times 27 \AA$. An acute step with five carbonate vacancies was created by subsequent removal of $\mathrm{CO}_{3}^{2-}$ groups followed by equilibration for $0.5 \mathrm{~ns}$ after each removal. The resulting surface charge density is $0.24 \mathrm{Cm}^{-2}$, which under experimental conditions corresponds to a zeta potential of about $-11 \mathrm{mV}$. The simulated surface step is thus in agreement with experimental conditions. The structure of this stepped surface slab is shown in Fig. 3, where it can be seen that the calcium ions neighboring the vacancies relax out of the surface and are found as adatoms on the surface.

Adsorption of both PAA and p-ASP occurred on this step during the simulation. Figs. 4 and 5 illustrate the simulation snapshots as well as the potential energy evolution for PAA and p-ASP respectively. As it can be seen PAA approaches the surface in its normal helix conformation before flattening out above the surface and then binding to surface Ca adatoms with four functional groups. In contrast, p-ASP approaches the surface with a terminal end, binding to the surface adatoms with three carboxy groups, the backbone of the molecule remaining upright. Binding takes place after about 170 ps for p-ASP whereas PAA takes about 400 ps to bind. p-ASP was found to systematically dissolve one of the surface $\mathrm{Ca}$ adatoms, which was not the case for PAA. On the plot of the potential energy as a function of time an energy barrier around 400 ps can be seen. This barrier occurs after the additive levels out over the surface, meaning it is associated with the binding and not the approach of the molecule. Most likely the barrier originates from the disruption of the surface water layers and the partial dehydration of the adatoms to 


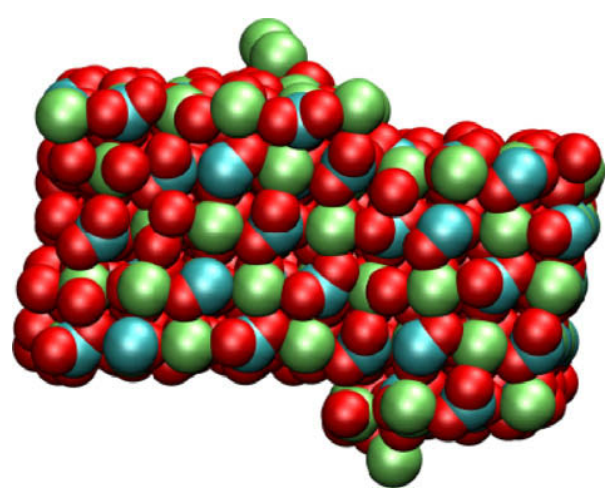

Fig. 3. Atomic structure of the defective acute step used in the simulations ( $\mathrm{Ca}=$ green, $\mathrm{C}=$ blue, $\mathrm{O}=\mathrm{red}$ ). (For interpretation of the references to color in this figure legend, the reader is referred to the web version of this article.)
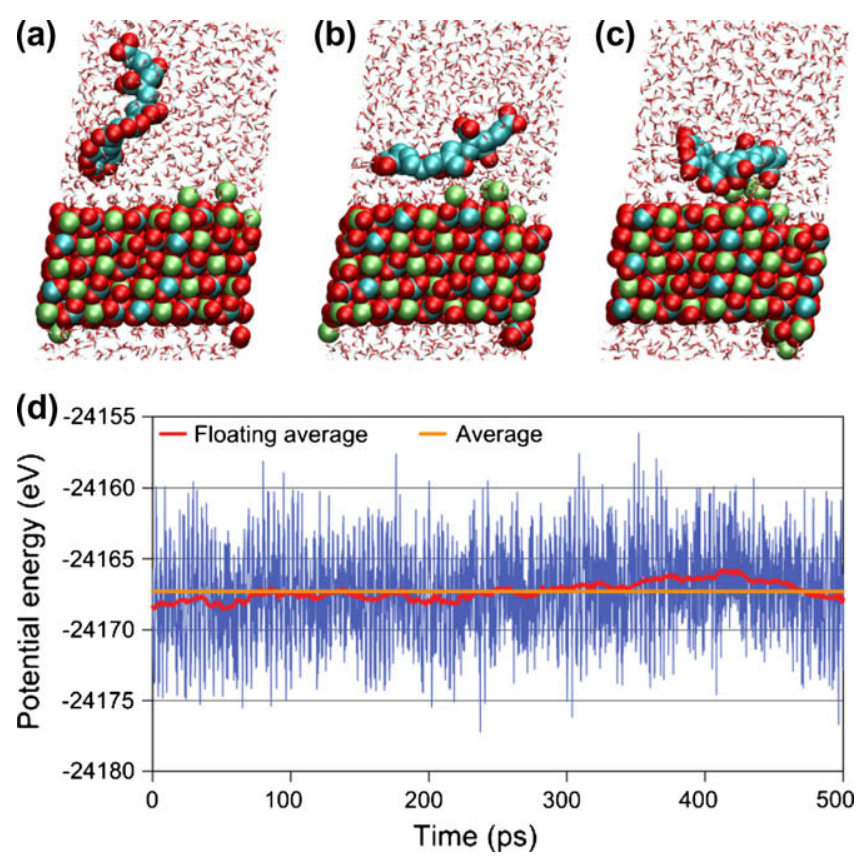

Fig. 4. Simulation snapshots of PAA at (a) 150 ps, (b) 400 ps and (c) 500 ps as well as $(\mathrm{d})$ the energy evolution of the simulation ( $\mathrm{Ca}=$ green, $\mathrm{C}=$ blue, $\mathrm{O}=\mathrm{red}$ ). (For interpretation of the references to color in this figure legend, the reader is referred to the web version of this article.)

which PAA binds. A similar barrier is not clearly visible for p-ASP where a number of energy maxima are visible most likely associated with the complex formations with the dissolved counter-ion. For both additives, the adsorption energies $E_{\text {ads }}$ were calculated according to following equation.

$$
\begin{aligned}
E_{\text {ads }} & =E_{\text {bound }}-E_{\text {unbound }} \\
& =E_{\text {bound }}-\left(E_{\text {surf +water }}+E_{\text {additive+water }}-E_{\text {water }}\right)
\end{aligned}
$$

$E_{\text {bound }}$ and $E_{\text {unbound }}$ are the potential energies of the states with the additive adsorbed and far from the surface respectively. Since the latter state is difficult to achieve in reasonable sized simulation cells, its energy can be expressed as the sum of the potential energies of the systems containing only the surface and water $E_{\text {surf+water }}$ and the additive and water $E_{\text {additive+water }}$ respectively, from which the self interaction energy of the doubly counted water $E_{\text {water }}$ has to be subtracted. For charged cells, where the interaction of charges in periodically repeated cells would result in infinitely large electrostatic self-interaction energies, the charge has been neutralized by
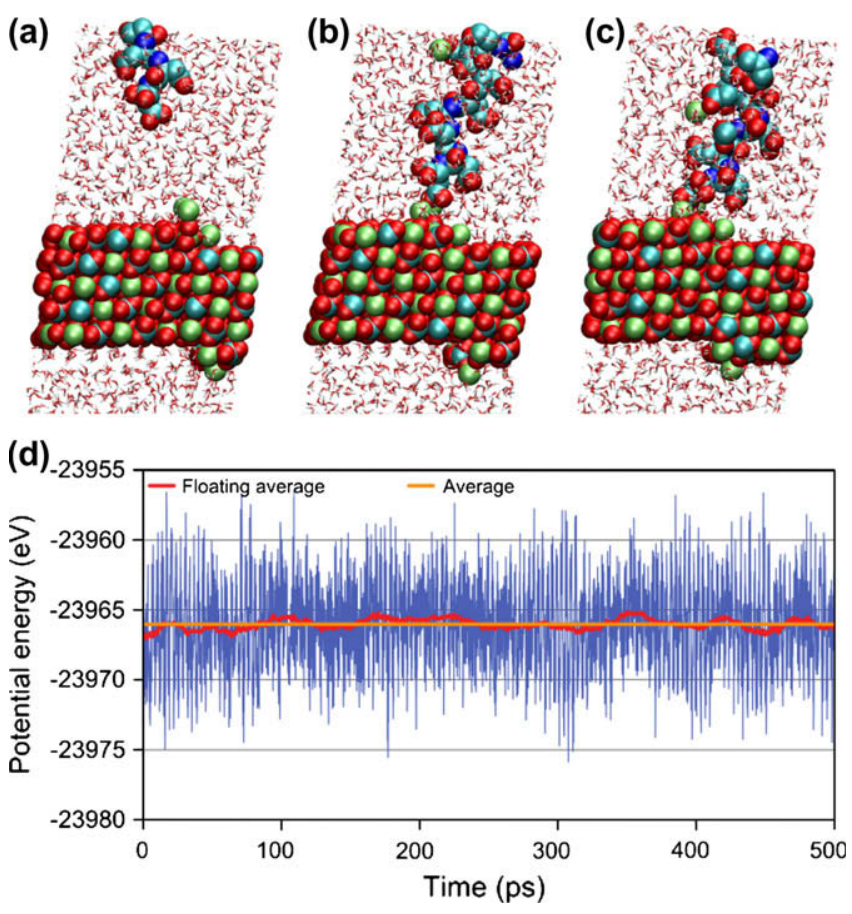

Fig. 5. Simulation snapshots of p-ASP at (a) 150 ps, (b) 400 ps and (c) 500 ps as well as $(\mathrm{d})$ the energy evolution of the simulation $(\mathrm{Ca}=$ green, $\mathrm{C}=$ light blue, $\mathrm{O}=$ red, $\mathrm{N}=$ dark blue). (For interpretation of the references to color in this figure legend, the reader is referred to the web version of this article.)

inserting a charge-balancing particle, the interaction of which with the rest of the simulation is eliminated by using a potential counterbalancing the electrostatic interaction.

The adsorption energy for PAA was calculated as $4.09 \mathrm{eV}$ (0.41 eV per carboxyl group) whereas for p-ASP $-13.04 \mathrm{eV}$ $(-1.3 \mathrm{eV}$ per carboxyl group) were obtained. The values per functional group are of the same order of magnitude as those presented in other studies [25,60-61] of the adsorption of small molecules on calcite surfaces. The positive value for PAA may seem surprising since the additive is seen to bind. A positive adsorption enthalpy for carboxylates on calcite has however also been measured experimentally [62-63]. The more favorable adsorption energy of p-ASP is explained by the negatively charged nitrogen on the backbone a result of the GAUSSIAN calculations-which result in a favorable electrostatic interaction with surface calcium ions as well the existence of an attractive van der Waals interaction between nitrogen and surface oxygen atoms.

Attempts at calculating reliable adsorption energies for short succinic acid (SA) and glutaric acid (GA) molecules proved to be difficult as these molecules are found to rapidly explore a manifold of configurations around the step, the time-window available for averaging of the potential energies becoming very short. Best possible results obtained are $-4.9 \mathrm{eV}$ for SA and $-2.7 \mathrm{eV}$ for GA. Nevertheless the rapid transition between configurations shows that short molecules most likely will not anchor to the surface and block growth sites for a time sufficiently long to noticeably affect growth.

The calculated adsorption energy is however solely the enthalpy part of the free energy. This means that PAA has to have a significant entropic contribution to the adsorption free energy, possibly explainable with the high flexibility of the singly bound backbone. Moreover an important part of the entropic contribution will also come from water molecules, where a gain in entropy is associated to going from a well defined surface layer to one disrupted by a polymer and also to the dehydration of the surface upon binding of a polymer. In order to get an idea of these effects the water density above the calcite surface has been calculated 

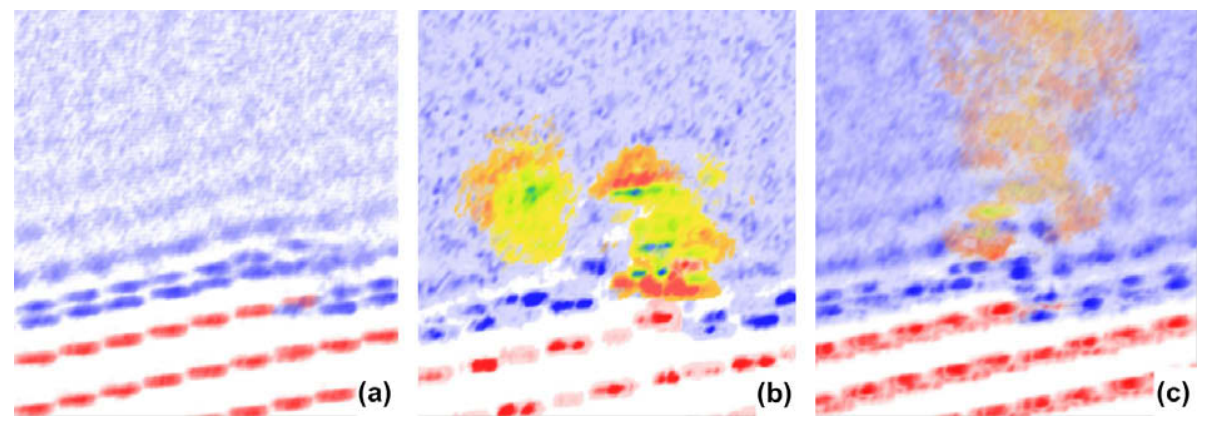

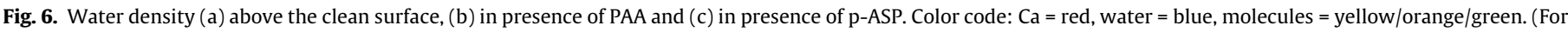
interpretation of the references to color in this figure legend, the reader is referred to the web version of this article.)

without and with presence of PAA and p-ASP, the results being shown in Fig. 6. As it can be seen a very well defined water structure of four high density layers exists above the clean surface (Fig. 6a). There is presence of water on a kink site, where the slab has lost calcium ions at the step edge. In presence of PAA (Fig. 6b) the water structure is severely disrupted, only the first and second layer being visible however much less well defined that without the additive. For p-ASP the water structure is better defined than for PAA, a number of consecutive layers being visible. Based on these results it seems that for PAA more water is released from the well defined low entropy surface sites and consequently a higher gain in entropy results for PAA than for p-ASP.

These calculations show that there are a number of differences between the two additives, which can explain the effects observed in experiment. On one hand PAA forms strong inner sphere complexes with the counter-ions in solution whereas p-ASP forms only outer sphere complexes with calcium ions. This means that the carboxy groups of PAA will be occupied by the counter-ions thus passivating this additive, whereas p-ASP functional groups will remain reactive. The difference in complexation is most likely due to the rigidity of the p-ASP peptide backbone, which makes it more difficult for two carboxy groups to complex with the same calcium ion. The difference is a lot less marked for sodium counter-ions where only a single functional group is needed to form the complex. This also explains the reduced yield observed in experiment with increasing PAA concentration by trapping of calcium ions, resulting even in a complete inhibition of precipitation at high polymer concentrations.

The adsorption energies for the two additives are very different with the p-ASP molecule showing a negative energy of adsorption whereas the PAA molecule has a positive energy component. This implies that p-ASP will bind on a much shorter time scale to the surface as attraction is stronger, which is also seen in the simulations where PAA took twice as long to reach the surface. Even though desorption could not be observed within the simulation-accessible timescale, the higher adsorption energy of p-ASP is expected to lead to a longer residence time of this additive at the surface leading to a more marked influence on crystal growth.

The conformation of the polymer at the surface differs for each molecule. PAA was observed to lie flat on the surface, whereas pASP remained upright. The steric repulsion between particles covered by an upright molecule will be stronger, preventing particle agglomeration and thus retaining the nano-sizes. This effect will however need to be further investigated using longer simulations at different additive and counter-ion concentrations.

\section{Conclusions}

Molecular dynamics simulations were used to explore the reasons for differences observations between seeded calcite crystals precipitated in the presence of two additives (PAA and p-ASP).
The simulations predict that adsorption is not favorable on flat perfect surfaces, but that an additional electrostatic driving force is necessary to overcome two main energy barriers. The energy barriers are attributed to the dehydration of the surface and the passage of the hydrophobic backbone through highly coordinated surface water layers. Simulations were thus run on surfaces presenting carbonate vacancies along acute steps. It was found that while both additives have the same functional groups, the backbone of the molecule could play an important role. On one hand its rigidity controls the complexation behavior of the additive with counter-ions in solution, making PAA less active for surface binding as it more easily forms stable complexes. The presence of nitrogen on the p-ASP backbone results in a higher attraction between the additive and the surface, resulting in a higher growth retarding effect. The conformation of the additive on the surface was also found to be different for the two additives, with the p-ASP molecule remaining upright and thus giving a higher steric barrier. This can prevent particle agglomeration and will retain nano sized particles throughout precipitation. The different conformations and the consequent disruption of the ordered water layer at the calcite crystal surface also contributed to different adsorption free energies, indicating a significant entropic contribution to the adsorption energy for the PAA.

These effects indicate a more rapid adsorption and longer residence times for the p-ASP at the calcite surface and thus can help explain the higher specific surface areas of calcite grown in presence of p-ASP compared to PAA. The knowledge gained from these simulations can be used to design next key experiments, which will provide guidelines for the specific design of additives which yield even higher specific surface area powders.

\section{Acknowledgments}

The authors are thankful to Prof. John Harding and Dr. Colin Freeman for helpful discussions. This work was supported by the European Sixth Framework Integrated Project Nanoker No. NMP3-CT-2005-515784.

\section{Appendix A. Supplementary material}

Supplementary data associated with this article can be found, in the online version, at doi:10.1016/j.jcis.2010.02.057.

\section{References}

[1] A.M. Belcher, X.H. Wu, R.J. Christensen, P.K. Hansma, G.D. Stucky, D.E. Morse, Nature 381 (1996) 56.

[2] A.W. Xu, Y.R. Ma, H. Cölfen, J. Mater. Chem. 17 (2007) 415.

[3] B. Wucher, W.B. Yue, A.N. Kulak, F.C. Meldrum, Chem. Mater. 19 (2007) 1111

[4] M.A. Osman, A. Atallah, U.W. Suter, Polymer 45 (2004) 1177.

[5] S. Wada, H. Suzuki, Tetrahedron Lett. 44 (2003) 399. 
[6] H. Cölfen, L.M. Qi, Chem A Eur J 7 (2001) 106.

[7] J. Rudloff, M. Antonietti, H. Cölfen, J. Pretula, K. Kaluzynski, S. Penczek Macromol. Chem. Phys. 203 (2002) 627.

[8] J.G. Yu, M. Lei, B. Cheng, X.J. Zhao, J. Solid State Chem. 177 (2004) 681.

[9] N. Wada, K. Kanamura, T. Umegaki, J. Colloid Interf. Sci. 233 (2001) 65.

[10] K. Westin, A.C. Rasmuson, J. Colloid Interf. Sci. 282 (2005) 359.

[11] M.M. Reddy, A.R. Hoch, J. Colloid Interf. Sci. 235 (2001) 365

[12] M.M. Reyhani, A. Oliveira, G.M. Parkinson, F. Jones, A.L. Rohl, M.I. Ogden, Int. J. Mod. Phys. B 16 (2002) 25.

[13] R.G. Jonasson, K. Rispler, B. Wiwchar, W.D. Gunter, Chem. Geol. 132 (1996) 215

[14] J.M. Didymus, S. Mann, W.J. Benton, I.R. Collins, Langmuir 11 (1995) 3130

[15] H.H. Teng, P.M. Dove, C.A. Orme, J.J. De Yoreo, Science 282 (1998) 724

[16] D. Volkmer, M. Fricke, T. Huber, N. Sewald, Chem. Commun. (2004) 1872.

[17] S. Elhadj, E.A. Salter, A. Wierzbicki, J.J. De Yoreo, N. Han, P.M. Dove, Cryst. Growth Des. 6 (2006) 197.

[18] J. Rieger, T. Frechen, G. Cox, W. Heckmann, C. Schmidt, J. Thieme, Faraday Discuss. 136 (2007) 265.

[19] U. Aschauer, J. Ebert, A. Aimable, P. Bowen, J. Colloid Interf. Sci. (submitted for publication).

[20] M. Donnet. Precipitation de $\mathrm{CaCO}_{3}$ en reacteur tubulaire: role des germes pour la controle de la granolumetrie et de la selectivité. Ph.D. Thesis, EPFL, Lausanne, 2002.

[21] J.O. Titiloye, S.C. Parker, S. Mann, J. Cryst. Growth 131 (1993) 533.

[22] M.A. Nygren, D.H. Gay, C.R.A. Catlow, M.P. Wilson, A.L. Rohl, J. Chem. Soc, Faraday Trans. 94 (1998) 3685.

[23] S.A. Ojo, B. Slater, C.R.A. Catlow, Mol. Simul. 28 (2002) 591.

[24] C.A. Orme, A. Noy, A. Wierzbicki, M.T. McBride, M. Grantham, H.H. Teng, P.M Dove, J.J. DeYoreo, Nature 411 (2001) 775.

[25] N.H. de Leeuw, T.G. Cooper, Cryst. Growth Des. 4 (2004) 123.

[26] D.M. Duffy, J.H. Harding, Langmuir 20 (2004) 7630.

[27] G.D. Bennett, J. Chem. Educ. 82 (2005) 1380.

[28] M. Born, K. Huang, Dynamical Theory of Crystal Lattices, Oxford University Press, Oxford, UK, 1954

[29] B.G. Dick, A.W. Overhauser, Phys. Rev. 112 (1958) 90.

[30] A. Pavese, M. Catti, G.D. Price, R.A. Jackson, Phys. Chem. Mineral. 19 (1992) 80

[31] A. Pavese, M. Catti, S.C. Parker, A. Wall, Phys. Chem. Mineral. 23 (1996) 89.

[32] N.H. de Leeuw, S.C. Parker, J. Phys. Chem. B 102 (1998) 2914.

[33] N.H. de Leeuw, S.C. Parker, J. Chem. Phys. 112 (2000) 4326.

[34] N.H. de Leeuw, S.C. Parker, Phys. Chem. Chem. Phys. 3 (2001) 3217.

[35] D.M. Duffy, J.H. Harding, J. Mater. Chem. 12 (2002) 3419.

[36] N.H. de Leeuw, J. Phys. Chem. B 106 (2002) 5241.

[37] S. Kerisit, S.C. Parker, J.H. Harding, J. Phys. Chem. B 107 (2003) 7676.

[38] G.W. Watson, E.T. Kelsey, N.H. deLeeuw, D.J. Harris, S.C. Parker, J. Chem. Soc Faraday Trans. 92 (1996) 433.

[39] N.H. de Leeuw, S.C. Parker, Phys. Rev. B 58 (1998) 13901.
[40] S. Kerisit, S.C. Parker, J. Am. Chem. Soc. 126 (2004) 10152.

[41] D. Spagnoli, D.J. Cooke, S. Kerisit, S.C. Parker, J. Mater. Chem. 16 (2006) 1997.

[42] S.L. Mayo, B.D. Olafson, W.A. Goddard, J. Phys. Chem. 94 (1990) 8897.

[43] K.P. Schröder, J. Sauer, M. Leslie, C.R.A. Catlow, J.M. Thomas, Chem. Phys. Lett. 188 (1992) 320.

[44] M.J. Frisch, G.W. Trucks, H.B. Schlegel, G.E. Scuseria, M.A. Robb, J.R. Cheeseman, J. Montgomery, J.A.T. Vreven, K.N. Kudin, J.C. Burant, J.M. Millam, S.S. Iyengar, J. Tomasi, V. Barone, B. Mennucci, M. Cossi, G. Scalmani, N. Rega, G.A. Petersson, H. Nakatsuji, M. Hada, M. Ehara, K. Toyota, R. Fukuda, J. Hasegawa, M. Ishida, T. Nakajima, Y. Honda, O. Kitao, H. Nakai, M. Klene, X. Li, J.E. Knox, H.P. Hratchian, J.B. Cross, V. Bakken, C. Adamo, J. Jaramillo, R. Gomperts, R.E. Stratmann, O. Yazyev, A.J. Austin, R. Cammi, C. Pomelli, J.W. Ochterski, P.Y. Ayala, K. Morokuma, G.A. Voth, P. Salvador, J.J. Dannenberg, V.G. Zakrzewski, S. Dapprich, A.D. Daniels, M.C. Strain, O. Farkas, D.K. Malick, A.D. Rabuck, K. Raghavachari, J.B. Foresman, J.V. Ortiz, Q. Cui, A.G. Baboul, S. Clifford, J. Cioslowski, B.B. Stefanov, G. Liu, A. Liashenko, P. Piskorz, I. Komaromi, R.L Martin, D.J. Fox, T. Keith, M.A. Al-Laham, C.Y. Peng, A. Nanayakkara, M. Challacombe, P.M.W. Gill, B. Johnson, W. Chen, M.W. Wong, C. Gonzalez, J.A. Pople, Gaussian, Inc., Wallingford, CT, 2004.

[45] R.S. Mulliken, J. Chem. Phys. 23 (1955) 1833.

[46] C.L. Freeman, J.H. Harding, D.J. Cooke, J.A. Elliott, J.S. Lardge, D.M. Duffy, J. Phys. Chem. C 111 (2007) 11943.

[47] W. Smith, T.R. Forester, J. Mol. Graph. 14 (1996) 136.

[48] F. Molnar, J. Rieger, Langmuir 21 (2005) 786.

[49] C.G. Sinn, R. Dimova, M. Antonietti, Macromolecules 37 (2004) 3444.

[50] P. Fenter, P. Geissbuhler, E. DiMasi, G. Srajer, L.B. Sorensen, N.C. Sturchio, Geochim. Cosmochim. Acta 64 (2000) 1221.

[51] R.E. Bulo, D. Donadio, A. Laio, F. Molnar, J. Rieger, M. Parrinello, Macromolecules 40 (2007) 3437.

[52] M.J. Yang, P.M. Rodger, J.H. Harding, S.L.S. Stipp, Mol. Simul. 35 (2009) 547.

[53] D. Spagnoli. Atomistic Simulations of the Mineral Water Interface. Ph.D. Thesis, University of Bath, Bath, UK, 2006.

[54] H.H. Teng, P.M. Dove, J.J. De Yoreo, Geochim. Cosmochim. Acta 64 (2000) 2255

[55] M. Donnet, A. Aimable, J. Lemaitre, P. Bowen, J. Phys. Chem. B (submitted for publication).

[56] D. Spagnoli, S. Kerisit, S.C. Parker, J. Cryst. Growth 294 (2006) 103.

[57] T.A. Kendall, S.T. Martin, J. Phys. Chem. A 111 (2007) 505.

[58] M. Donnet, P. Bowen, J. Lemaitre, J. Colloid Interf. Sci. 340 (2009).

[59] D.F. Evans, H. Wennerström, The Colloidal Domain, Wiley-VCH, New York, 1999.

[60] C.L. Freeman, I. Asteriadis, M.J. Yang, J.H. Harding, J. Phys. Chem. C 113 (2009) 3666.

[61] M. Yang, J. Harding, S.L.S. Stipp, Mineral. Mag. 72 (2008) 295

[62] M.R. Böhmer, Y.E. Sofi, A. Foissy, J. Colloid Interf. Sci. 164 (1994) 126.

[63] J. Plank, G, Bassioni, Zeitschrift Fur Naturforschung Section B-a J. Chem. Sci. 62 (2007) 1277. 